\title{
Strategies to Improve Delivery of Cirrhosis Care
}

\author{
Akshata Moghe, MD PhD \\ Vera Yakovchenko, $\mathrm{MPH}^{2}$ \\ Timothy Morgan, $M D^{3,4}$ \\ Heather McCurdy, MSN NP-C ${ }^{5}$ \\ Dawn Scott, MS PA-C ${ }^{6}$ \\ Karine Rozenberg-Ben-Dror, PharmD ${ }^{7}$ \\ Shari Rogal, MD MPH ${ }^{8,9, *}{ }^{\circ}$
}

\author{
Address \\ ${ }^{1}$ Department of Medicine, Division of Gastroenterology, Hepatology, and Nutri- \\ tion, University of Pittsburgh Medical Center, Pittsburgh, PA, USA \\ ${ }^{2}$ Center for Healthcare Organization and Implementation Research, Edith Nourse \\ Rogers Memorial VA Hospital, Bedford, MA, USA \\ ${ }^{3}$ Gastroenterology Section, VA Long Beach Healthcare System, Long Beach, CA, USA \\ ${ }^{4}$ Division of Gastroenterology, Department of Medicine, University of California, \\ Irvine, CA, USA \\ ${ }^{5}$ VA Ann Arbor Healthcare System, Ann Arbor, MI, USA \\ ${ }^{6}$ Central Texas Veterans Healthcare System, Temple, TX, USA \\ ${ }^{7}$ Veteran Affairs Great Lakes Health Care System, VISN 12 PBM, Westchester, IL, USA \\ ${ }^{*}, 8$ Center for Health Equity Research and Promotion, VA Pittsburgh Healthcare \\ System, Research Office Building (151R), University Drive C, Pittsburgh, PA, \\ 15240, USA \\ Email: Shari.Rogal@va.gov \\ ${ }^{9}$ Departments of Medicine and Surgery, University of Pittsburgh, Pittsburgh, PA, USA
}

Published online: 24 May 2021

(C) This is a U.S. government work and not under copyright protection in the U.S.; foreign copyright protection may apply 2021

This article is part of the Topical Collection on Liver

Keywords Care delivery $\cdot$ Implementation · Ascites · Encephalopathy $\cdot$ Hospitalization $\cdot$ Readmission

\section{Abstract}

Purpose of review This review provides an overview of the current state of research around improving healthcare delivery for patients with cirrhosis in the outpatient, inpatient, and transitional care settings.

Recent findings Recent studies have broadly employed changes to the model of care delivery, team composition, and technology to improve cirrhosis care. In the outpatient setting, approaches have included engaging caregivers, patient navigators, and nonphysicians and using virtual care, smartphone applications, and wearables. Inpatient care approaches have focused on the role of interdisciplinary teams, education interventions, and changes to the medical record system, while post-discharge interventions have 
included day hospitals and care coordinator interventions. This review also describes the Veterans Health Administration's novel, population-level approach to delivery of cirrhosis care, and addressed how the pandemic has impacted the delivery of cirrhosis care. Summary Comprehensive, evidence-based approaches to delivering high-quality cirrhosis care continue to evolve to meet the needs of a growing population in an ever-changing healthcare environment.

\section{Introduction}

Cirrhosis is a leading cause of global morbidity and mortality, accounting for 40,000 deaths, 150,000 hospitalizations, and four billion dollars in healthcare costs annually in the USA alone [1]. Although there are established, evidence-based practices associated with improved outcomes for patients with cirrhosis, the quality of cirrhosis care varies widely. In fact, the majority of patients with cirrhosis do not receive evidence-based care [2]. For example, a recent study found that adherence to HCC surveillance guidelines was followed in only approximately $30 \%$ of patients with cirrhosis [2]. While there are many known barriers to the delivery of cirrhosis care, recent progress has been made in understanding and addressing these barriers.

In an important step toward improving cirrhosis care, the American Association for the Study of
Liver Diseases (AASLD) developed and disseminated standard, evidence-based recommendations for quality measures $[3 \bullet \bullet]$. These measures broadly focus on preventing and managing complications of cirrhosis, addressing the high readmission rates for patients hospitalized for hepatic decompensation, and measuring and improving patientreported outcomes. Beyond these efforts to define high-quality care, a growing number of investigators and healthcare systems have developed novel approaches to cirrhosis care delivery. The aim of this review was to assess the recent strategies to improve the delivery of cirrhosis care across the outpatient, inpatient, and transitional care settings.

\section{Methods}

\section{Results}

We conducted a scoping review of strategies to improve cirrhosis care published between 2015 and 2020. We searched Google Scholar, PubMed, and Clinicaltrials.gov using terms ["cirrhosis" or "advanced liver disease"] AND ["quality improvement" or "strategies to improve care" or "interventions"]. The findings were then limited to articles in English that pertained to management of cirrhosis and its complications. Articles were excluded if they focused on transplantation and were then organized by practice location, including outpatient, inpatient, and transitional care settings (Fig. 1).

\section{Advances in outpatient care delivery}

Recent work has pushed the boundaries beyond traditional one-on-one, faceto-face doctor-patient care in the outpatient specialty clinic setting for patients with cirrhosis (Table 1). Such studies have examined alternate care delivery models and challenge traditional views of providers. A recent study focusing on the role of advanced practice providers (APPs) examined a large American 


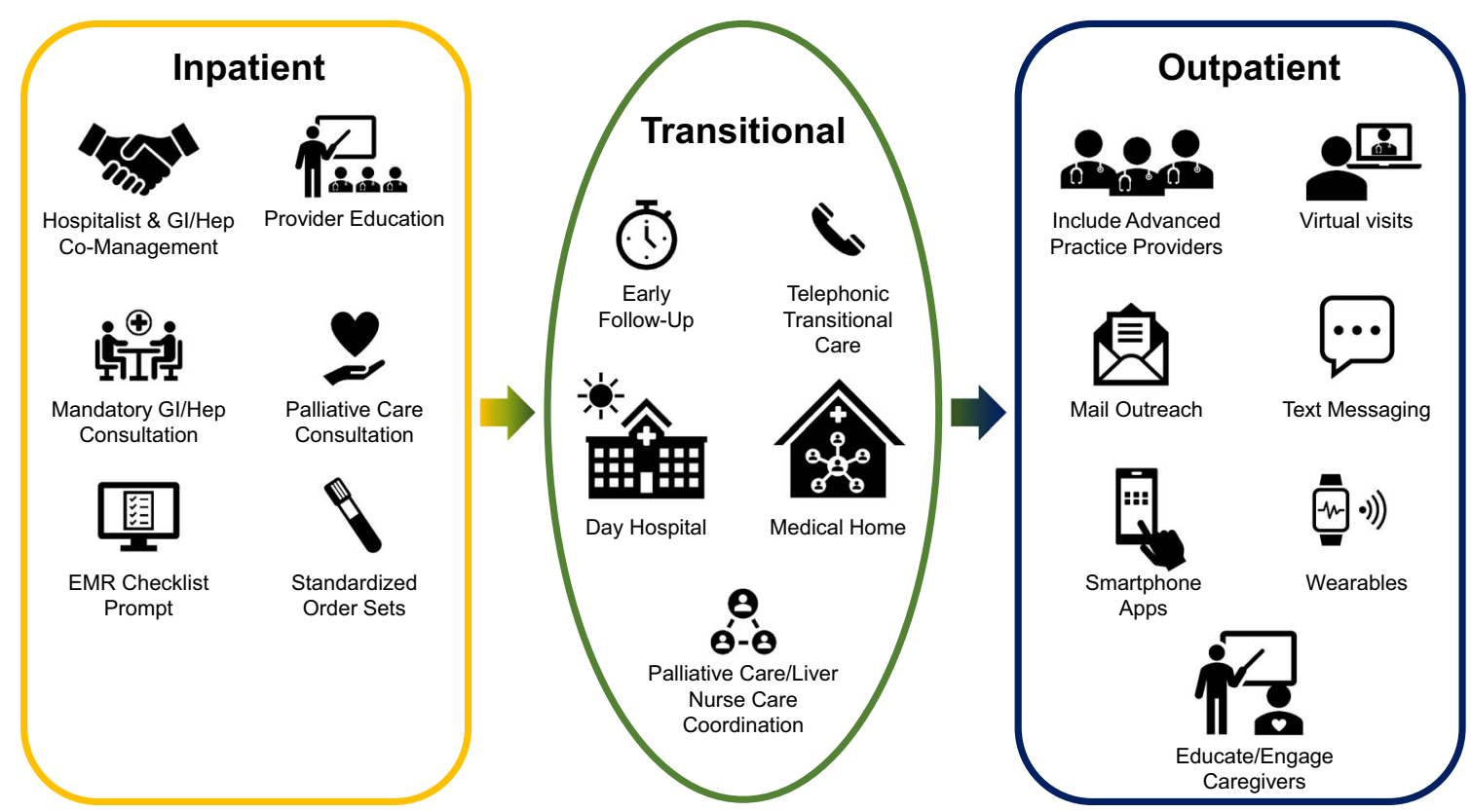

Fig. 1. Interventions to improve the delivery of cirrhosis care

cohort of patients with cirrhosis and found that including APPs on the care team for patients with cirrhosis was associated with increased HCC screening, rifaximin use in hepatic encephalopathy, and variceal screening, along with reduced 30-day readmission rates. Patients under the combined care of APPs and gastroenterologists or hepatologists fared the best and had better care than those seen by physicians alone $[4 \bullet \bullet]$. Another approach focused on engaging caregivers in care through an education intervention and found improved encephalopathy outcomes [5]. Yet another study found that mailed outreach with and without a patient navigator significantly improved HCC screening for patients with cirrhosis [6].

Beyond changing the model of who delivers care, the ways in which care is being delivered are also changing. Many recent interventions focus on providerand patient-facing technology. Prior to the ubiquitous use of virtual care during the SARS-Co-V2 epidemic, Su et al. found a survival benefit for veterans who received virtual liver disease care through the Specialty Care Access NetworkExtension of Community Healthcare Outcome (VA SCAN-ECHO) project $[7 \bullet \bullet]$. The SCAN-ECHO model at the VA functions to provide consultations via telemedicine to physicians in underserved areas. This model has also been successfully utilized for the treatment of hepatitis $\mathrm{C}$ in other settings and countries $[8,9]$.

Likewise, there is growing evidence that mobile phone text-messaging and other technology-based remote monitoring systems can improve care across a number of chronic diseases [10, 11]. Smartphone applications (apps) such as the EncephalApp_Stroop app have been demonstrated to be effective and reliable in the diagnosis of minimal and covert encephalopathy $[12,13]$. The Patient Buddy App, which monitors medication use, patient weight, and daily sodium intake and performs cognitive and fall-risk assessments, was associated with reduced 
Table 1. Summary of successful cirrhosis care delivery interventions by setting

\begin{tabular}{|c|c|c|}
\hline \multirow[t]{2}{*}{ Setting } & Approach & \\
\hline & Provider and care model interventions & Technology-based approaches \\
\hline \multirow[t]{5}{*}{ Outpatient } & $\begin{array}{l}\text { Advanced practice provider care } \\
\text {-increased HCC and variceal surveillance }[4 \bullet \bullet] \\
\text {-decreased readmission }[4 \bullet \bullet] \\
\text {-improved survival }[4 \bullet \bullet]\end{array}$ & $\begin{array}{l}\text { Virtual visits } \\
\text {-improved survival }[7 \bullet \bullet] \\
\text { Mobile text-messaging } \\
\text {-improved medication adherence }[10]\end{array}$ \\
\hline & Caregiver education & \\
\hline & -improved encephalopathy care [5] & $\begin{array}{l}\text { Smartphone applications } \\
\text {-improved encephalopathy management [12-14] }\end{array}$ \\
\hline & $\begin{array}{l}\text { Mailed patient reminders } \\
\text {-increased HCC surveillance [6] }\end{array}$ & -acceptable to patients with ascites [15] \\
\hline & & $\begin{array}{l}\text { Wearables } \\
\text {-improved encephalopathy monitoring [16] } \\
\text {-improved physical performance [17] }\end{array}$ \\
\hline \multirow[t]{6}{*}{ Inpatient } & $\begin{array}{l}\text { Hospitalist/GI co-management service } \\
\text {-trend towards improved survival and decreased } \\
\text { readmission [18] }\end{array}$ & $\begin{array}{l}\text { Medical record checklist } \\
\text {-decreased readmission [23] }\end{array}$ \\
\hline & $\begin{array}{l}\text { Automatic specialty consultation } \\
\text {-improved quality indicators (but not readmission) [19] }\end{array}$ & $\begin{array}{l}\text { Order sets } \\
\text {-decreased readmission [22-24] }\end{array}$ \\
\hline & $\begin{array}{l}\text { Palliative care consultation } \\
\text {-decreased readmission }[21 \bullet]\end{array}$ & \\
\hline & Education interventions & \\
\hline & $\begin{array}{l}\text {-decreased readmission and longer "time to tap" for } \\
\text { patients with ascites }[25,26]\end{array}$ & \\
\hline & -decreased encephalopathy [20] & \\
\hline \multirow[t]{3}{*}{ Transitional Care } & $\begin{array}{l}\text { Early follow-up } \\
\text {-improved survival [29] }\end{array}$ & \\
\hline & $\begin{array}{l}\text { Telephonic transitional care } \\
\text {-improved survival [30] }\end{array}$ & \\
\hline & $\begin{array}{l}\text { Day hospital } \\
\text {-decreased readmission }[31 \bullet] \\
\text {-improved survival }[31 \bullet]\end{array}$ & \\
\hline
\end{tabular}


encephalopathy-related admissions [14]. Similarly, an application-based ascites management intervention, which included a scale and Bluetooth connection between the app and the electronic health record, found that the app was acceptable to patients and providers and resulted in frequent, weight-based diuretic adjustments [15]. Wearable technologies are currently being trialed for monitoring encephalopathy [16] and addressing frailty [17]. Thus, outpatient care delivery evidence has supported tech-based approaches and engaging expanding upon who is engaged in the cirrhosis care team.

\section{Advances in inpatient care delivery}

Several models of inpatient care have been evaluated. Consultation with hepatology has been associated with improvements in the delivery of guideline-adherent care, but not necessarily decreased readmission [18, 19]. Although mandatory gastroenterology consultation improved quality indicators such as early endoscopy for patients with variceal bleeding, it did not decrease readmission [19]. Specialty inpatient care, using specialists to provide primary inpatient care and tighter co-management models, are also gaining traction [20]. Among patients admitted with spontaneous bacterial peritonitis (SBP), those co-managed by hospitalists and hepatologists together had a trend towards improved survival, reduced intensive care unit transfers, and lower 30day readmissions compared to patients managed by hospitalists with hepatology consultation [18]. In this study, the co-management model was associated with more timely paracentesis $(75 \%$ vs. $43 \%, p=0.05)$, increased albumin use ( $97 \%$ vs. $65 \%, p=0.002)$, and a significantly higher number of patients being discharged on SBP prophylaxis $(91 \%$ vs. $37 \%, p<0.001)$ [18]. In another approach, inpatient palliative care consultation for patients admitted with decompensated cirrhosis was associated with decreased readmission and increased out-of-hospital time [21•].

Recent evidence also supports streamlining inpatient cirrhosis care using electronic health record solutions. Using standardized order sets can leverage the electronic medical record (EMR) system and can reduce provider burden compared to paper checklists [22]. Implementation of an electronic order set for cirrhosis was associated with significantly reduced 30-day readmissions when compared with a hand-held checklist [23]. Similarly, electronic prompts for SBP prophylaxis at discharge was associated with significantly decrease 30-day readmissions [23]. An upcoming trial will test order set efficacy and implementation in an eight hospital settings in Canada [24].

Recent provider education interventions have focused on improving adherence to guideline-based care. Rawson et al. assessed the impact of education on "time from admission to ascites tap." The intervention included an informational booklet and a didactic session about SBP for house staff. The intervention was associated with significant improvement on a knowledge quiz as well as significantly reduced time to tap [25]. A similar educational intervention for medical residents caring for patients with ascites was shown to reduce readmission rates [26]. Education of discharging providers, patients, and family members regarding appropriate use and titration of lactulose is thought to be the most important intervention to prevent future episodes of HE [20]. Thus, education interventions, electronic solutions, and changes to the structures of inpatient care and the care team were proven useful in the inpatient setting. 


\section{Advances in transitional care delivery}

Transitional care delivery focuses on improving post-hospitalization outcomes with the

primary goal of reducing readmission. A key recent advance in this area is the focus on distinguishing "preventable readmissions" from other readmissions. Tapper et al. recently advocated for a more nuanced approach to defining preventable admissions resulting from low-quality care (e.g., not providing antibiotic prophylaxis for gastrointestinal bleeding) vs. necessary or unpreventable admissions, whose delay results in morbidity and mortality [20]. With that in mind, recent studies have focused on assessing risk factors for early readmission, which could ultimately help target high-risk patients in need of intervention. Mumtaz et al. reviewed the Nationwide Readmission Database and developed the "Mumtaz readmission risk score" which includes patient age, insurance/payer, etiology of cirrhosis and complications, comorbidities, paracentesis, hemodialysis, and disposition at discharge [27]. This validated scoring system helped classify patients into low $(<20 \%)$, medium $(20-30 \%)$, and high (>30\%) risk of readmission at 30 days [27]. Similarly, Gaspar et al. developed predictive models for readmission and 180-day mortality using factors associated with readmission and mortality, respectively [28]. Such models could be used to guide directed interventions.

Early outpatient follow-up after discharge has been associated with reduced mortality and, in some studies, with reduced readmission rates. The optimal setting and content for this follow-up is being evaluated. In a 13-year retrospective study of veterans with cirrhosis, Kanwal and colleagues found that follow-up within 7 days of discharge was associated with significantly reduced mortality $(3.2 \%$ vs. $5.2 \%, \mathrm{HR}=0.6)$ but also increased readmission $(15.3 \%$ vs. $13.8 \%, \mathrm{HR}=$ 1.10) [29]. Similarly, another study found that a 30-day care coordinator intervention using outpatient telephonic transitional care was associated with decreased mortality but not readmission [30]. In contrast, the HEPACONTROL randomized control trial, conducted in Spain, randomized patients to usual care vs. follow-up in a hepatology unit "day hospital" within 7 days of discharge [31 $\bullet$. The day hospital provided care until patients were deemed stable and provided outpatients with real-time radiology and lab testing. The intervention patients had significantly reduced readmission rates, post-discharge emergency department visits, and mortality compared to patients receiving standard outpatient followup [31•]. A similar care-coordinator-led study was piloted in the UK and is undergoing further evaluation. This intervention provides patients with nurse specialists who provide case management and support, liaising between patients, families, and providers for up to 6 months [32]. In addition, the nurse offers patients and their caregivers' financial, social, and psychological support and more information about their illness. Finally, the nurse also supports community professionals involved in the care of the patient by providing information on best practice and anticipatory care planning. The intervention was deemed feasible and was well-received by patients, caregivers, and healthcare providers, and further trials are ongoing [32]. Another trial is similarly investigating the concept of a "Cirrhosis Medical Home" for patients' post-hospital discharge [33]. Thus, a number of approaches aim to define the optimal post-discharge care model to prevent readmissions for patients with cirrhosis. 
The Veterans Health Administration (VHA) cares for the largest cohort of patients with cirrhosis in the USA. VHA's Liver Disease Program is a leading example of a comprehensive approach to improving the delivery of cirrhosis care. In 2015, to proactively address the population of veterans with chronic liver diseases and improve care processes across VHA hospitals, VHA developed a national learning collaborative, the hepatic innovation team (HIT) [34-37]. The HIT has been centered around lean quality improvement and system redesign and run by a centralized leadership team. HITs are organized by region and consist of clinicians, system redesign experts, and a coordinator. These regional teams meet regularly with the centralized leadership team.

Each year, regional teams submit proposals for their annual goals and activities and receive salary support that is used to fund part of the time of the coordinators. Teams meet regularly with the leadership, engage in collaborative planning, and help to contribute to best practices, which helps facilities with less resources to improve care $[35,36]$. The HIT collaborative is responsible for a regular educational series, collaborative planning meetings, and education around quality improvement methods.

To support the work of the HIT, a dashboard development team with expertise in data analysis, population management, and liver disease was assembled to develop national population management tools, including hepatitis $\mathrm{C}$, hepatitis B, and advanced liver disease dashboards, to identify and address gaps in care for veterans with liver disease.

The Liver Disease Program is developing and piloting several innovative QI initiatives. These include an approach to systematically assessing veteran-reported outcomes, such as symptoms, care satisfaction, and quality of life. Additionally, VHA data indicate that like outside of VHA, many patients have unrecognized or undiagnosed cirrhosis. The Liver Disease Program is also piloting a protocol to use administrative data to identify these veterans and bring them in for further evaluation to establish a cirrhosis diagnosis and link these veterans to highquality cirrhosis care. VHA is also supporting an intensive implementation intervention to help VHA facilities with opportunities to improve cirrhosis care [38]. This stepped-wedge, cluster randomized trial is evaluating the use of data-driven implementation strategies for cirrhosis care. VHA's Liver Disease Program also develops, updates, and disseminates educational resources, including VHAspecific guidelines for managing hepatitis $\mathrm{C}$, cirrhosis, and fatty liver disease. Thus, VHA has developed a comprehensive, national, population-based approach to improve the quality of cirrhosis care across the system.

The COVID-19 pandemic has had a profound impact on the delivery of cirrhosis care across all settings, necessitating proactive innovation to maintain high-quality care. The pandemic has resulted in changed approaches to screening and assessment methods and the approach to and delivery of inpatient care [39]. For example, there has been a focus on avoiding hospitalizations and other scarce resource utilization via deferring non-urgent testing, substituting with less-resource intensive testing, and implementing telehealth [40]. While disruptions in preventative care can result in a cascade of negative downstream impacts, the pandemic has also necessitated rapid innovation [40, 41]. Rapid 


\section{Conclusions}

development and dissemination of guidance regarding the management of patients with cirrhosis during the COVID-19 pandemic have loosened HCC surveillance recommendations and suggesting that prophylactic beta-blockers can be used in patients with evidence of portal hypertension, to avoid endoscopy $[42 \bullet \bullet, 43]$. Telehealth has emerged as the predominant form of outpatient care during the pandemic, and studies have illustrated that this approach can help address disparities in access to and quality of care related to age, race, socioeconomic status, and health literacy $[42 \bullet \bullet, 44]$. It remains to be seen if the rapid acceleration of telemedicine implementation spurred on by the pandemic may change the paradigm of cirrhosis care delivery even after the pandemic is over [45]. However, certainly the pandemic has and will continue to pose both challenges and opportunities to reconstruct cirrhosis care delivery.

Interventions to improve the quality of cirrhosis care delivery have included patient-, provider- and system-facing approaches. These approaches have varied in the scope of their focus, from individual quality metrics (e.g., increasing antibiotic prophylaxis) to overhauling the way that care is delivered (e.g., medical home models). A recent theme across this evolving body of work is a focus on using technology to improve care. However, more data are needed to understand the necessary implementation supports that lead to the delivery of high-value, high-quality cirrhosis care. Steps to improving the quality of cirrhosis care depend on having measurable outcomes to track, the ability to track them, and a systematic approach to implementing quality improvement interventions.

\section{Declarations}

\section{Conflict of interest}

The work surrounding this publication is in compliance with Ethical Standards. All authors completed a Conflict of Interest form. Dr. Shari Rogal reports grants from VA Partnered Evaluation Initiative, the NIH, and the VA HIV Hepatitis and Related Conditions Program Office during the conduct of the study. Dr. Timothy Morgan reports grants from AbbVie, Merck, Genfit, and Gilead outside the submitted work. Dr. Akshata Moghe, Dr. Karine Rozenberg-Ben-Dror, Vera Yakovchenko, Dawn Scott, and Heather McCurdy declare no conflict of interests. This material is based upon work supported by the Department of Veterans Affairs, Veterans Health Administration, and Office of Research and Development. The views expressed in this article are those of the authors and do not necessarily reflect the position or policy of the Department of Veterans Affairs or the United States government.

\section{Human and Animal Rights and Informed}

This article does not contain any studies with human or animal subjects performed by any of the authors. 
Springer Nature remains neutral with regard to jurisdictional claims in published maps and institutional affiliations.

1. Kim WR, Brown RS Jr, Terrault NA, El-Serag H. Burden of liver disease in the United States: summary of a workshop. Hepatology. 2002;36(1):227-42. https:// doi.org/10.1053/jhep.2002.34734.

2. Kardashian A, Patel AA, Aby ES, Cusumano VT, Soroudi C, Winters AC, et al. Identifying quality gaps in preventive care for outpatients with cirrhosis within a large, academic health care system. Hepatol Commun. 2020;4(12):1802-11. https://doi.org/10.1002/hep4. 1594.

3.• Kanwal F, Tapper EB, Ho C, Asrani SK, Ovchinsky N, Poterucha J, et al. Development of quality measures in cirrhosis by the practice metrics committee of the American Association for the Study of Liver Diseases. Hepatology. 2019;69(4):1787-97. https://doi.org/10. 1002/hep.30489

This guidance provides standard definitions of quality measures that can be used as the backbone of quality improvement programs.

4.• Tapper EB, Hao S, Lin M, Mafi JN, McCurdy H, Parikh $\mathrm{ND}$, et al. The quality and outcomes of care provided to patients with cirrhosis by advanced practice providers. Hepatology. 2020;71(1):225-34. https://doi.org/10. 1002/hep.30695

This study demonstrated the importance of advanced practice providers in the delivery of high-quality cirrhosis care.

5. Rosenblatt R, Yeh J, Gaglio PJ. Long-term management: modern measures to prevent readmission in patients with hepatic encephalopathy. Clin Liver Dis. 2020;24(2):277-90. https://doi.org/10.1016/j.cld. 2020.01.007.

6. Singal AG, Tiro JA, Marrero JA, McCallister K, Mejias C, Adamson B, et al. Mailed outreach program increases ultrasound screening of patients with cirrhosis for hepatocellular carcinoma. Gastroenterology. 2017;152(3):608-1.

e4. https://doi.org/10.1053/j.gastro.2016.10.042.

7.• Su GL, Glass L, Tapper EB, Van T, Waljee AK, Sales AE. Virtual consultations through the veterans administration SCAN-ECHO project improves survival for veterans with liver disease. Hepatology.

2018;68(6):2317-24. https://doi.org/10.1002/hep. 30074

This study demonstrated that virtual care consulation resulted in improved survival for Veterans with liver disease.

8. Stotts MJ, Grischkan JA, Khungar V. Improving cirrhosis care: The potential for telemedicine and mobile health technologies. World J Gastroenterol. 2019;25(29):3849-56. https://doi.org/10.3748/wjg. v25.i29.3849.

9. Marciano S, Haddad L, Plazzotta F, Mauro E, Terraza S, Arora S, et al. Implementation of the $\operatorname{ECHO}((\mathrm{R}))$ telementoring model for the treatment of patients with hepatitis C. J Med Virol. 2017;89(4):660-4. https:// doi.org/10.1002/jmv.24668.

10. Thakkar J, Kurup R, Laba TL, Santo K, Thiagalingam A, Rodgers A, et al. Mobile telephone text messaging for medication adherence in chronic disease: a meta-analysis. JAMA Intern Med. 2016;176(3):340-9. https:// doi.org/10.1001/jamainternmed.2015.7667.

11. Yakovchenko V, Hogan TP, Houston TK, Richardson L, Lipschitz J, Petrakis BA, et al. Automated text messaging with patients in department of veterans affairs specialty clinics: cluster randomized trial. J Med Internet Res. 2019;21(8):e14750. https://doi.org/10.2196/14750.

12. Bajaj JS, Thacker LR, Heuman DM, Fuchs M, Sterling RK, Sanyal AJ, et al. The stroop smartphone application is a short and valid method to screen for minimal hepatic encephalopathy. Hepatology. 2013;58(3):1122-32. https://doi.org/10.1002/hep. 26309.

13. Bajaj JS, Heuman DM, Sterling RK, Sanyal AJ, Siddiqui M, Matherly S, et al. Validation of EncephalApp, smartphone-based stroop test, for the diagnosis of covert hepatic encephalopathy. Clin Gastroenterol Hepatol. 2015;13(10):1828-3.

e1. https://doi.org/10.1016/j.cgh.2014.05.011.

14. Ganapathy D, Acharya C, Lachar J, Patidar K, Sterling $\mathrm{RK}$, White $\mathrm{MB}$, et al. The patient buddy app can potentially prevent hepatic encephalopathy-related readmissions. Liver Int. 2017;37(12):1843-51. https:// doi.org/10.1111/liv.13494.

15. Bloom P, Wang T, Marx M, Tagerman M, Green B, Arvind A, et al. A smartphone app to manage cirrhotic ascites among outpatients: feasibility study. JMIR Med Inform. 2020;8(9):e17770. https://doi.org/10.2196/ 17770.

16. Zhang QWP, Liu Y, Peng B, Zhou Y, Zhou Z, et al. A real-time wireless wearable electroencephalography system based on Support Vector Machine for encephalopathy daily monitoring. Int J Distrib Sens Netw. 2018;14:1-9.

17. Duarte-Rojo A, Bloomer PM, Rogers RJ, Hassan MA, Dunn MA, Tevar AD, et al. Introducing EL-FIT (Exercise and Liver FITness): a smartphone app to prehabilitate and monitor liver transplant candidates. Liver Transpl. 2020. https://doi.org/10.1002/lt.25950.

18. Desai AP, Satoskar R, Appannagari A, Reddy KG, Te HS, Reau N, et al. Co-management between hospitalist and hepatologist improves the quality of care of inpatients with chronic liver disease. J Clin Gastroenterol. 2014;48(4):e30-6. https://doi.org/10.1097/MCG. ob013e3182a87f70. 
19. Ghaoui R, Friderici J, Desilets DJ, Lagu T, Visintainer P, Belo A, et al. Outcomes associated with a mandatory gastroenterology consultation to improve the quality of care of patients hospitalized with decompensated cirrhosis. J Hosp Med. 2015;10(4):236-41. https://doi. org/10.1002/jhm.2314.

20. Tapper EB. Tackling 30-day readmissions in patients with cirrhosis. Curr Hepatol Rep. 2018;17:8-14.

21• Shinall MC Jr, Karlekar M, Martin S, Gatto CL, Misra S, Chung CY, et al. COMPASS: a pilot trial of an early palliative care intervention for patients with end-stage liver disease. J Pain Symptom Manag. 2019;58(4):614-622 e3. https://doi.org/10.1016/j. jpainsymman.2019.06.023

This research has started to change the paradigm of palliative care utilization for patients with cirrhosis.

22. Tapper EB. Building effective quality improvement programs for liver disease: a systematic review of quality improvement initiatives. Clin Gastroenterol Hepatol. 2016;14(9):1256-65 e3. https://doi.org/10. 1016/j.cgh.2016.04.020.

23. Tapper EB, Finkelstein D, Mittleman MA, Piatkowski G, Chang M, Lai M. A quality improvement initiative reduces 30-day rate of readmission for patients with cirrhosis. Clin Gastroenterol Hepatol. 2016;14(5):753-9. https://doi.org/10.1016/j.cgh. 2015.08.041.

24. Carbonneau M, Eboreime EA, Hyde A, CampbellScherer D, Faris P, Gramlich L, et al. The cirrhosis care Alberta (CCAB) protocol: implementing an evidencebased best practice order set for the management of liver cirrhosis - a hybrid type I effectivenessimplementation trial. BMC Health Serv Res. 2020;20(1):558. https://doi.org/10.1186/s12913-02005427-8.

25. Rawson TM, Bouri S, Allen C, Ferreira-Martins J, Yusuf A, Stafford N, et al. Improving the management of spontaneous bacterial peritonitis in cirrhotic patients: assessment of an intervention in trainee doctors. Clin Med (Lond). 2015;15(5):426-30. https://doi.org/10. 7861/clinmedicine.15-5-426.

26. Lim N, Sanchez O, Olson A. Impact on 30-d readmissions for cirrhotic patients with ascites after an educational intervention: a pilot study. World J Hepatol. 2019;11(10):701-9. https://doi.org/10. 4254/wjh.v11.i10.701.

27. Mumtaz K, Issak A, Porter K, Kelly S, Hanje J, Michaels $\mathrm{AJ}$, et al. Validation of risk score in predicting early readmissions in decompensated cirrhotic patients: a model based on the administrative database. Hepatology. 2019;70(2):630-9. https://doi.org/10. 1002/hep.30274.

28. Gaspar R, Rodrigues S, Silva M, Costa-Moreira P, Morais R, Andrade P, et al. Predictive models of mortality and hospital readmission of patients with decompensated liver cirrhosis. Dig Liver Dis.
2019;51(10):1423-9. https://doi.org/10.1016/j.dld. 2019.03.016.

29. Kanwal F, Asch SM, Kramer JR, Cao Y, Asrani S, El-Serag HB. Early outpatient follow-up and 30-day outcomes in patients hospitalized with cirrhosis. Hepatology. 2016;64(2):569-81. https://doi.org/10.1002/hep. 28558.

30. Rao BB, Sobotka A, Lopez R, Romero-Marrero C, Carey W. Outpatient telephonic transitional care after hospital discharge improves survival in cirrhotic patients. World J Hepatol. 2019;11(8):646-55. https://doi.org/ 10.4254/wjh.v11.i8.646.

31. Morales BP, Planas R, Bartoli R, Morillas RM, Sala M, Casas I, et al. HEPACONTROL. A program that reduces early readmissions, mortality at 60 days, and healthcare costs in decompensated cirrhosis. Dig Liver Dis. 2018;50(1):76-83. https://doi.org/10.1016/j. dld.2017.08.024

This publication provides information about a successful intervention in the post-discharge period.

32. Kimbell B, Murray SA, Byrne H, Baird A, Hayes PC, MacGilchrist A, et al. Palliative care for people with advanced liver disease: a feasibility trial of a supportive care liver nurse specialist. Palliat Med. 2018;32(5):919-29. https://doi.org/10.1177/ 0269216318760441.

33. Cirrhosis Medical Home (CMH). ClinicalTrialsgov Identifier: NCT04581369.

34. Belperio PS, Chartier M, Gonzalez RI, Park AM, Ross DB, Morgan TR, et al. Hepatitis C care in the Department of Veterans Affairs: building a foundation for success. Infect Dis Clin N Am. 2018;32(2):281-92. https://doi.org/10.1016/j.idc.2018.02.011.

35. Park A, Gonzalez R, Chartier M, Rogal S, Yakovchenko $\mathrm{V}$, Ross D, et al. Screening and treating hepatitis C in the VA: achieving excellence using lean and system redesign. Fed Pract. 2018;35(7):24-9.

36. Rogal SS, Yakovchenko V, Waltz TJ, Powell BJ, Gonzalez R, Park A, et al. Longitudinal assessment of the association between implementation strategy use and the uptake of hepatitis C treatment: Year 2. Implement Sci. 2019;14(1):36. https://doi.org/10.1186/ s13012-019-0881-7.

37. Rogal SS, Yakovchenko V, Waltz TJ, Powell BJ, Kirchner JE, Proctor EK, et al. The association between implementation strategy use and the uptake of hepatitis $\mathrm{C}$ treatment in a national sample. Implement Sci. 2017;12(1):60. https://doi.org/10.1186/s13012-0170588-6.

38. Rogal SS, Yakovchenko V, Morgan T, Bajaj JS, Gonzalez R, Park A, et al. Getting to implementation: a protocol for a Hybrid III stepped wedge cluster randomized evaluation of using data-driven implementation strategies to improve cirrhosis care for Veterans. Implement Sci. 2020;15(1):92. https://doi.org/10.1186/s13012020-01050-7. 
39. Mahmud N, Hubbard RA, Kaplan DE, Serper M. Declining cirrhosis hospitalizations in the wake of the COVID-19 pandemic: a national cohort study. Gastroenterology. 2020;159(3):1134-.

e3. https://doi.org/10.1053/j.gastro.2020.05.005.

40. Tapper EB, Asrani SK. The COVID-19 pandemic will have a long-lasting impact on the quality of cirrhosis care. J Hepatol. 2020;73(2):441-5. https://doi.org/10. 1016/j.jhep.2020.04.005.

41. Serper M, Shaked A, Olthoff KM, Hoteit M, Appolo B, Reddy KR. A local response to COVID-19 for advanced liver disease: current model of care, challenges and opportunities. J Hepatol. 2020;73(3):708-9. https:// doi.org/10.1016/j.jhep.2020.05.022.

42••. Fix OK, Hameed B, Fontana RJ, Kwok RM, McGuire $\mathrm{BM}$, Mulligan DC, et al. Clinical best practice advice for hepatology and liver transplant providers during the COVID-19 pandemic: AASLD expert panel consensus statement. Hepatology. 2020;72(1):287304. https://doi.org/10.1002/hep.31281

This paper gives real-world advice for managing care delivery during the COVID-19 pandemic.
43. Boettler T, Newsome PN, Mondelli MU, Maticic M, Cordero E, Cornberg M, et al. Care of patients with liver disease during the COVID-19 pandemic: EASL-

ESCMID position paper. JHEP Rep. 2020;2(3):100113. https://doi.org/10.1016/j.jhepr.2020.100113.

44. GD MN, Kaplan D, Serper M. Major shifts in outpatient cirrhosis care delivery attributable to the COVID-19 pandemic: a national cohort study. Hepatol Commun. 2020. https://doi.org/10.1002/hep4.1638.

45. Serper M, Cubell AW, Deleener ME, Casher TK, Rosenberg DJ, Whitebloom D, et al. Telemedicine in liver disease and beyond: can the COVID-19 crisis lead to action? Hepatology. 2020;72(2):723-8. https://doi. org/10.1002/hep.31276.

\section{Publisher's Note}

Springer Nature remains neutral with regard to jurisdictional claims in published maps and institutional affiliations. 